\title{
Uso de sistemas rotatórios no tratamento endodôntico em odontopediatria
}

\author{
Use of rotary systems in endodontic treatment in dental pediatrics \\ Uso de sistemas rotativos en tratamiento endodóntico en pediatría dental
}

Recebido: 29/11/2021 | Revisado: 07/12/2021 | Aceito: 09/12/2021 | Publicado: 17/12/2021

\author{
Mariana Karlla de Melo Lins \\ ORCID: https://orcid.org/0000-0002-3360-3209 \\ Centro Universitário Mário Pontes Jucá, Brasil \\ E-mail: Marilinsmelo@ hotmail.com \\ Adilson Rodrigues da Silva Filho \\ ORCID: https://orcid.org/0000-0003-2752-873X \\ Centro Universitário Mário Pontes Jucá, Brasil \\ E-mail: adilson.rodrigues.14@ hotmail.com \\ Ana Maria Guerra Costa \\ ORCID: https://orcid.org/0000-0002-6564-4718 \\ Centro Universitário Mário Pontes Jucá, Brasil \\ E-mail: anaguerracosta@yahoo.com.br
}

\begin{abstract}
Resumo
Esta revisão de literatura objetivou avaliar o uso dos sistemas de limas rotatórias em comparação com os sistemas padrões de limas manuais, levando em consideração o tempo e a qualidade do preparo de canais radiculares em dentes decíduos, bem como o efeito do tratamento com essas limas na cooperação e no desenvolvimento de dor pósoperatória nas crianças. O levantamento bibliográfico foi baseado em artigos publicados nos últimos 5 anos, encontrados nas seguintes bases de dados da área da saúde: PubMed/Medline, Scopus, LILACS, SciELO e Cocrhane. Os resultados demonstraram que tratamentos endodônticos com limas rotatórias exibiram menor tempo e melhor qualidade do preparo biomecânimo dos canais em dentes decíduos, bem como resultaram em menor experiência de dor pósóperatória, quando comparado ao uso de limas manuais. Com base nisso, é possível concluir que pulpectomias realizadas com limas rotatórias são eficazes, seguras e diminuem o tempo de tratamento e a dor pós-operatória no tratamento endodôntico pediátrico.
\end{abstract}

Palavras-chave: Saúde da criança; Odontopediatria; Pulpectomia; Preparo de canal radicular.

\begin{abstract}
This literature review aimed to evaluate the use of rotary file systems compared to standard manual file systems, taking into account the time and quality of root canal preparation in primary teeth, as well as the effect of treatment with these files on cooperation and the development of postoperative pain in children. The bibliographic survey was based on articles published in the last 5 years, found in the following databases in the health area: PubMed/Medline, Scopus, LILACS, SciELO and Cocrhane. The results showed that endodontic treatments with rotary files exhibited shorter time and better quality of the biomechanical preparation of the canals in primary teeth, as well as resulted in less experience of postoperative pain, when compared to the use of manual files. Based on this, it is possible to conclude that pulpectomies performed with rotary files are effective, safe and reduce treatment time and postoperative pain in pediatric endodontic treatment.
\end{abstract}

Keywords: Child health; Pediatric dentistry; Pulpectomy; Root canal preparation.

\section{Resumen}

Esta revisión de la literatura tuvo como objetivo evaluar el uso de sistemas de limas rotativas en comparación con los sistemas de limas manuales estándar, teniendo en cuenta el tiempo y la calidad de la preparación del conducto radicular en los dientes temporales, así como el efecto del tratamiento con estas limas en la cooperación y el desarrollo de dolor postoperatorio en niños. La encuesta bibliográfica se basó en artículos publicados en los últimos 5 años, encontrados en las siguientes bases de datos del área de salud: PubMed / Medline, Scopus, LILACS, SciELO y Cocrhane. Los resultados mostraron que los tratamientos de endodoncia con limas rotativas exhibieron menor tiempo y mejor calidad de la preparación biomecánica de los conductos en los dientes temporales, además de resultar en una menor experiencia de dolor postoperatorio, en comparación con el uso de limas manuales. En base a esto, es posible concluir que las pulpectomías realizadas con limas rotativas son efectivas, seguras y reducen el tiempo de tratamiento y el dolor postoperatorio en el tratamiento endodóntico pediátrico.

Palabras clave: Salud del niño; Odontología pediátrica; Pulpectomía; Preparación del conducto radicular. 


\section{Introdução}

A saúde bucal oral é imprescindível para manter a qualidade de vida de um indivíduo. A primeira infância é um período de muito risco a problemas bucais, principalmente para o desenvolvimento de cáries dentárias, que são altamente incidentes durante a infância. A odontologia atual preza pela conservação do elemento dentário decíduo quando for possível, pois esses dentes preservam o comprimento do arco dentário, guiam a erupção dos dentes permanentes, auxiliam na mastigação, na prevenção de movimentos anormais da língua e na fonética. Dessa forma, o procedimento adotado irá depender da extensão das lesões cariosas, mas sempre com o objetivo de manter o elemento dentário e evitar a sintomatologia dolorosa do paciente. Nos casos de lesões com comprometimento pulpar, torna-se necessário intervenções pulpares, como capeamento pulpar, pulpotomia, que consiste na retirada da porção coronal da polpa, ou a pulpectomia, que retira toda a polpa dentária. (Natchiyar et al., 2021; Pawar et al., 2021).

A pulpectomia é considerada o tratamento de escolha em casos de pulpites irreversíveis, garantindo a integridade dos tecidos orais e dos dentes, livre de sintomatologia, até a esfoliação natural do dente decíduo para a irrupção do permanente. Esse procedimento compreende a remoção completa da polpa dentária, a limpeza desses canais, o preparo do canal através de sua instrumentação com limas endodônticas, e a obturação do canal com material reabsorvível. Entretanto, as pulpectomias realizadas em dentes decíduos representam um grande desafio para os cirurgiões-dentistas, principalmente por causa da anatomia radicular complexa e do seu processo fisiológico de reabsorção, além do fator comportamental da criança, que nem sempre é cooperativa com o tratamento, e a experiência de dor pós-operatória, que pode ser traumática para essas crianças e ainda prejudicar a execução dos procedimentos clínicos. Desse modo, são fatores que devem ser levados em consideração durante o tratamento endodôntico. (Ahmed et al., 2013; Chugh et al., 2021; Natchiyar et al., 2021).

Com base nisso, além dos fatores comportamentais ligados diretamente à primeira infância, o sucesso clínico do tratamento endodôntico dependerá crucialmente do preparo biomecânico dos canais radiculares, pois a limpeza e a modelagem dos canais irão permitir a remoção do tecido infectado e fornecer acesso para a irrigação completa dos canais, concedendo um ambiente propício para a adequada obturação dos canais. O procedimento de instrumentação dos canais é que permite o preparo biomecânico, sendo executado através de soluções irrigantes e limas endodônticas. A instrumentação realizada com limas manuais é amplamente utilizada dentro da odontopediatria. Porém, esses sistemas de limas manuais são limitados quanto à cooperação das crianças, pois consome um maior tempo clínico durante o tratamento. Pensando nisso, outros sistemas de limas foram desenvolvidos ao longo do tempo, com o objetivo de melhorar o processo clínico das pulpectomias. (Chugh et al., 2021; George et al., 2016; Manchanda et al., 2020).

Os sistemas de limas rotatórias vêm se difundindo atualmente como uma alternativa ao uso das limas manuais, permitindo instrumentações mais rápidas e com maior conforto para o paciente. As limas rotatórias são bastante difundidas em tratamentos endodônticos de dentes permanentes, e o seu uso na odontopediatria também está em crescimento, sendo desenvolvidos sistemas rotatórios específicos para dentes decíduos. Porém, ainda são necessários estudos que levem em consideração o uso dessas limas rotatórias nos elementos dentários decíduos, avaliando os efeitos da redução do tempo de instrumentação na qualidade do preparo e obturação dos canais, além das vantagens no condicionamento comportamental e na experiência de dor das crianças. (Ahmed et al., 2013; Chauhan et al., 2019; Barasuol et al., 2021).

Levando em consideração as ponderações acima, o objetivo deste trabalho é avaliar o uso dos sistemas de limas rotatórias em comparação com os sistemas padrões de limas manuais, levando em consideração o tempo e a qualidade do preparo de canais radiculares em dentes decíduos, bem como o efeito do tratamento com essas limas na cooperação e no desenvolvimento de dor pós-operatória nas crianças. 


\section{Metodologia}

Este trabalho foi desenvolvido utilizando como guia as diretrizes para revisões literárias do PRISMA. (Moher et al., 2009; Hermont et al., 2021). Para isso, antes de iniciar a busca por estudos, foram traçados os critérios a serem utilizados durante a seleção. Dessa forma, os critérios de inclusão para esta revisão foram: artigos publicados entre janeiro de 2015 e junho de 2021; estudos escritos na língua portuguesa, inglesa e espanhola; artigos que abordem o uso de instrumentos endodônticos rotatórios; estudos que foquem no preparo do canal radicular de dentes decíduos; trabalhos que abordem o pósoperatório de tratamentos com as limas rotatórias. Já os critérios de exclusão incluíram: estudos não disponíveis integralmente para leitura; revisões de literatura; trabalhos que abordem somente o uso de limas endodônticas manuais para pulpectomias em dentes decíduos; artigos que estudem somente outras características do preparo de canais radiculares, sem demonstrar o papel das limas nesse procedimento; abordar tratamento ou procedimentos realizados somente em dentes permanentes.

Utilizando-se desses critérios, foi realizada uma busca eletrônica dentro das seguintes bases de dados da área da saúde: PubMed/Medline, Scopus, LILACS, SciELO e Cochrane. Com isso, a busca dos artigos foi efetuada através da seguinte estratégia elaborada com base nos seguitnes descritores Decs/ MeSH, e termos alternativos unidos a operadores booleanos: "Pediatric Dentistry" AND "Pulpectomy" AND "Rotary Endodontics". Dessa forma, o levantamento bibliográfico inicial foi feito com base na leitura dos títulos e resumos dos artigos, selecionando os que atenderam aos critérios estabelecidos anteriormente. Após isso, uma pesquisa manual das referências dos estudos presentes nas bases de dados foi feita, para complementar a seleção dos artigos. No fim, foram excluídos os estudos duplicados e os que não se encaixavam no perfil de critérios. Os artigos finais foram analisados a partir de uma tabela de resumo, para auxiliar a transcrição dos dados de cada estudo.

\section{Resultados}

A busca eletrônica dos artigos nas bases de artigos resultou em 67 estudos disponíveis dentro da data de publicação estabelecida nas bases de dados selecionadas. Com a leitura inicial dos títulos e resumos, restaram 16 artigos que faziam parte da área de interesse para esta revisão. Deste número, 01 artigo foi removido por estar duplicado entre as bases de dados, restando um total de 15 artigos para a avaliação completa de seu conteúdo. A busca manual das referências resultou em mais um artigo de interesse. Dessa forma, restaram um total de 16 artigos que estavam dentro dos critérios de seleção e foram avaliados para integrar a revisão. As etapas de seleção dos artigos estão ilustradas em um fluxograma adaptado a partir das diretrizes do PRISMA (Figura 1). 
Figura 1 - Fluxograma adaptado do PRISMA statement for systematic reviews

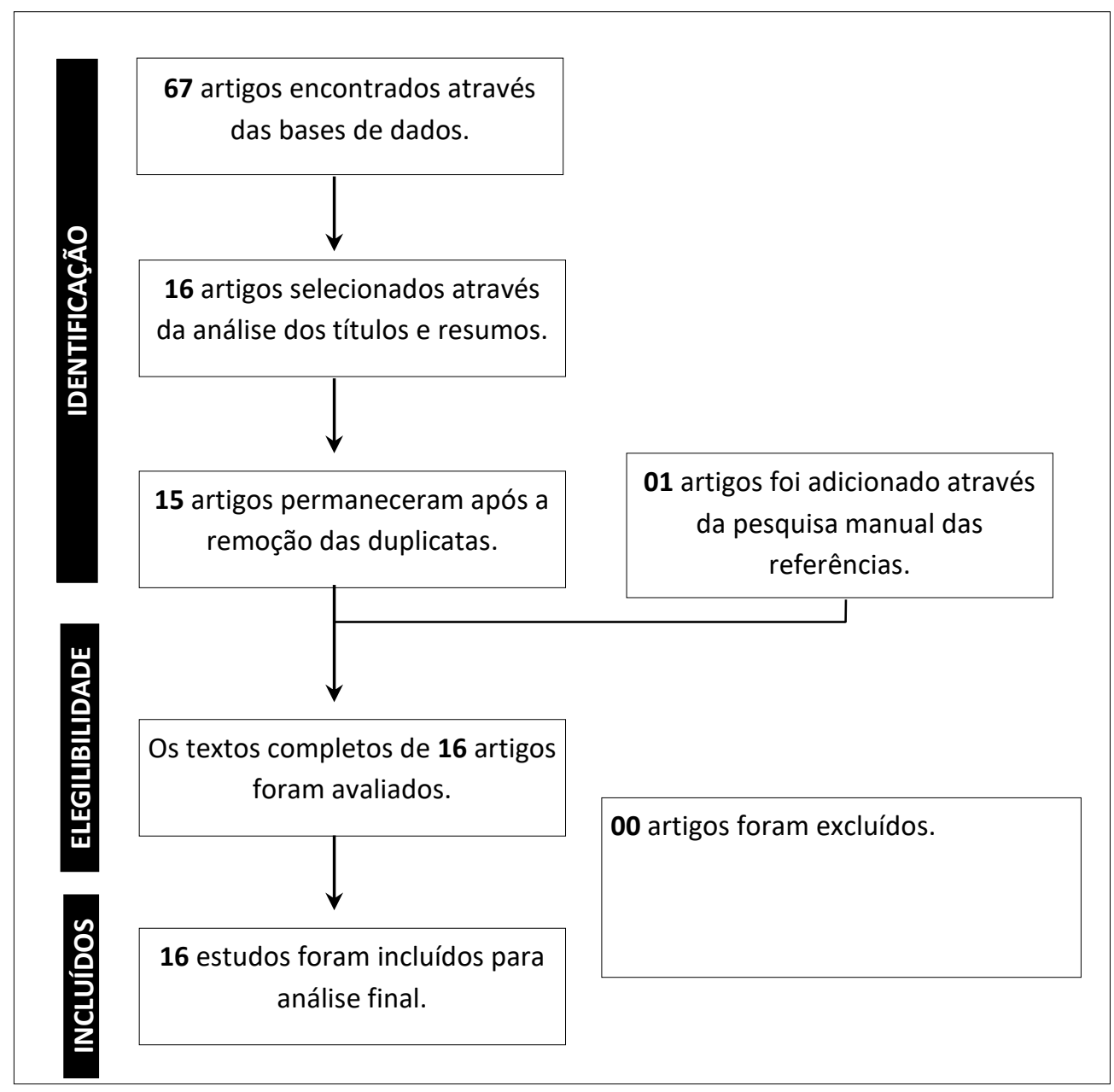

Fonte: Moher et al. (2009).

Um resumo com as informações contidas nos artigos selecionados está disposto na tabela 1 deste trabalho. O primeiro estudo, de Barasoul et al., (2021), comparou o tempo de preparação químico-mecânica dos canais, o comportamento da criança, a dor pós-operatória e o uso de medicação após o tratamento endodôntico de crianças com base em limas manuais e limas rotativas. Com isso, os dados demonstraram que o tempo médio para a preparação químico-mecânica em limas manuais foi de 24,5 minutos, enquanto o tempo médio para limas rotatórias foi de 17 minutos, diminuindo o tempo médio em 7,6 minutos. Todavia, nenhuma diferença estatística foi achada em relação ao comportamento da criança durante a preparação dos canais, nem em relação ao limite de obturação apical, a presença de dor e uso de medicação pós-operatória entre as limas manuais e as rotatórias.

Outro estudo avaliou o uso clínico de sistemas de limas rotatórias e limas manuais na instrumentação de dentes decíduos. Boonchoo et al., (2020) descobriram que o tempo de instrumentação dos canais era maior quando usadas limas manuais, com uma média de 7,38 minutos, enquanto as limas rotatórias realizavam o procedimento em 3,23 minutos, com uma diminuição de 4 minutos do procedimento. Não foram encontradas diferenças entre o tempo de obturação dos canais em relação às duas técnicas, sendo que, o preenchimento desses canais apresentou uma melhor qualidade entre os elementos tratados com limas rotatórias. Apesar disso, não houve diferenças em relação ao sucesso do tratamento clínico e radiográfico entre 6 e 12 meses após o tratamento.

Anteriormente, Govindaraju et al., (2017) também avaliaram a qualidade de instrumentação e obturação, bem como o tempo para realizar o tratamento entre limas manuais e dois sistemas de limas rotatórias. Em relação à qualidade de obturação 
dos canais, os dentes tratados com limas manuais tiveram preenchimento ideal em $60 \%$ dos canais mesiais e $40 \%$ dos distais. Já os dois sistemas de limas rotatórias apresentaram preenchimento ideal em 73,3\% e 60\% dos canais mesiais, e 60\% e 53,3\% dos canais distais. Não há diferença estatística entre a qualidade de obturação dos canais entre as limas manuais e rotatórias, mas, de modo geral, a qualidade de obturação dos canais mesiais foram superiores aos canais distais. Também foi comprovada uma diferença significativa entre o tempo de instrumentação dos três sistemas de limas, sendo as limas rotatórias as que apresentaram os menores tempos de uso.

De modo semelhante, Jeevanandan e Thomas, (2018) realizaram um estudo comparando a preparação do canal radicular e o tempo de instrumentação em molares decíduos instrumentados com limas manuais e rotatórias, através de tomografias computadorizadas. Canais tratados com instrumentos manuais exibiram maior volume do canal após a instrumentação do que os rotatórios. Já em relação ao tempo de instrumentação, as limas manuais apresentaram a maior média de instrumentação, com 194,5 segundos, apresentando diferenças significativas em relação às limas rotatórias.

Já Jeevanandan et al., (2020) avaliaram o nível de dor pós-operatória entre tratamentos de canais com limas manuais e rotatórias. Os dados mostraram que, no intervalo entre 6 e 12 após o tratamento endodôntico, a intensidade de dor foi maior nos pacientes tratados com limas manuais, sendo relatada uma redução gradual na intensidade da dor durante as 12 primeiras horas, quando comparado às 6 primeiras horas pós-tratamento. Após 24 horas, não existiam mais diferenças entre a intensidade de dor entre os tipos de limas utilizadas.

Um ano depois, Jeevanandan et al., (2021) avaliaram a qualidade de obturação e a presença de dor pós-operatória em obturações em canais de molares decíduos feitas com limas manuais e rotatórias. Sobre a obturação dos canais, foi possível notar uma melhor qualidade entre os dentes tratados através de limas rotatórias. A avaliação de dor pós-operatória comprovou menor intensidade de dor em casos tratados com limas rotatórias entre o $1^{\circ}, 3^{\circ}$ e $4^{\circ}$ dias pós-tratamento, diminuindo gradualmente a dor entre o $5^{\circ}$ e $6^{\circ}$ dias, não apresentando mais diferenças de dor entre as limas manuais e rotatórias. Mesmo com uma menor percepção de dor após tratamentos com limas rotatórias, não foi observada redução de dor durante o primeiro dia, independente da técnica utilizada para o tratamento. Sabendo disso, todos os pacientes experimentaram dor pós-operatória nos primeiros dois dias independente da qualidade de obturação dos canais. Entretanto, do $3^{\circ}$ ao $5^{\circ}$ dias após o tratamento, os pacientes com obturação adequada dos canais já não sentiam mais dor, mas os demais pacientes ainda relatavam dor leve a moderada. Dessa forma, $50 \%$ das crianças com obturação ideal dos canais não experimentou dor pós-operatória, enquanto $72,8 \%$ dos casos sem obturação ideal experimentou dor de leve a moderada após o tratamento.

Por sua vez, Lakshmanan et al., (2020) avaliaram a qualidade de instrumentação e obturação de canais radiculares tratados com limas rotatórias e manuais. O tempo médio de instrumentação foi menor em tratamentos realizados com limas rotatórias, com valor de 75,6 segundos. Já as outras técnicas manuais tiveram tempo médio de instrumentação de 95 segundos. Sobre a qualidade de obturação dos canais, tratamentos realizados com limas rotatórias tiveram canais com obturação ideal em $60 \%$ dos casos, e os casos tratados com limas manuais obtiveram sucesso em $27 \%$ dos canais.

Em seguida, o trabalho de Mehlawat et al., (2019) realizou uma avaliação do tempo de instrumentação e da eficácia de limpeza em canais de molares decíduos com limas rotatórias e manuais. O tempo médio da preparação biomecânica dos canais foi de 4,32 minutos quando usadas limas manuais, e 3,54 minutos quando foi usado limas rotatórias, sendo assim, o sistema de limas com menor tempo de preparação do canal. A eficácia da limpeza dos canais foi avaliada em terços. Assim, o terço coronal dos canais apresentou uma pontuação média de eficácia mais baixa em dentes tratados com limas rotatórias do que em manuais. Já o terço médio do canal apresentou melhor média de pontuação para as limas rotatórias, ocorrendo o mesmo quando avaliado o terço apical dos canais. Porém, apesar dessas diferenças de pontuação na limpeza dos canais, não houve diferenças estatisticamente significativas entre os dois tipos de lima. 
Por sua vez, Nair et al., (2018) avaliaram a dor pós-operatória após tratamento endodôntico em molares decíduos com limas manuais e dois sistemas de limas rotatórias. Os dados demonstram que 44\% dos pacientes tratados com o sistema de limas manuais apresentaram dor moderada após 6 horas do tratamento, diminuindo para 32\% após as 12 horas. Após as 24 primeiras horas do tratamento, somente $4 \%$ desses pacientes ainda relataram algum tipo de dor. Os que realizaram o procedimento através de limas rotatórias não relatam incidência e dor pós-operatória.

Mais tarde, Pawar et al., (2021) compararam o tempo de instrumentação e a qualidade da obturação em tratamentos endodônticos realizados por limas rotatórias e manuais em dentes decíduos. Em relação ao tempo de instrumentação, foi demonstrado que os dois sistemas de limas rotatórias levaram em média 10,9 e 14,8 minutos, enquanto o sistema de limas manuais teve um tempo médio de instrumentação em 19,9 minutos. Dessa forma, limas rotatórias foram associadas a um menor tempo de instrumentação dos canais, já que ocorreu uma redução de $20 \%$ do tempo entre os sistemas de limas. Sobre a qualidade de obturação desses canais, os resultados mostraram que os sistemas de limas rotatórias apresentaram um maior número de obturações de canais ideais em relação aos sistemas manuais.

Anteriormente, Ramazani et al., (2016) realizara um estudo para avaliar a eficácia de limpeza e modelagem de canais radiculares e o tempo de instrumentação com limas rotatórias e manuais em molares decíduos. Os dados ilustraram que limas rotatórias possuem uma eficácia de limpeza dos canais melhor do que as limas manuais. Essas limas também demonstraram uma excelente modelagem dos canais, bem como foram o sistema de limas com menor tempo de instrumentação dentre os avaliados nesse estudo.

O estudo de Rathi et al., (2021) quantificou os detritos extrusados e a eficácia de limpeza durante a preparação de canais radiculares em dentes decíduos com sistemas de limas rotatórias. Todas as técnicas de instrumentação com limas rotatórias causaram uma quantidade significativa de detritos extrusados, acarretando no chamado "efeito taper lock", onde a lima fica envolvida na região coronal do canal, não permitindo que os detritos da instrumentação saiam do canal, empurrando esses detritos para a porção apical do canal. Já a eficácia de instrumentação foi considerada melhor nos terços coronal e, principalmente, no médio, sendo a região apical a de menor eficiência de instrumentação.

Por sua vez, Seema et al., (2020) avaliaram a remoção de dentina e o estreitamento do preparo do canal feito com limas manuais e dois sistemas de limas rotatórias. Ambas as limas, manual e rotatória, apresentaram uma quantidade média semelhante de remoção de dentina, com exceção da superfície mesial de dentes tratados com limas manuais, que apresentaram maior remoção de dentina no terço coronal da raiz. A conicidade do preparo dos canais demonstrou que tanto as limas manuais quanto as rotatórias produziam uma boa redução do canal radicular, não existindo diferenças significativas entre os valores obtidos.

Por outro lado, Shah et al., (2021) compararam o tempo de instrumentação, o tempo e a qualidade de obturação entre sistemas de limas manuais e rotatórias em pulpectomias de molares decíduos. Limas manuais possuíram um maior tempo médio necessário para a instrumentação, com 27,87 minutos, e os dois outros sistemas de limas rotatórias apresentaram tempo médio de 19,25 minutos e 21,89 minutos. De modo semelhante, as limas manuais exibiram um maior tempo de obturação dos canais, com uma média de 5,44 minutos, enquanto os sistemas rotatórios levaram em média 4,71 e 4,62 minutos para a obturação. Em relação à qualidade da obturação, não houve diferenças significativas entre as limas manuais e as rotatórias com base na extensão do preenchimento do canal e a presença ou ausência de vazios.

Previamente, Srinivas et al., (2019) realizaram um estudo para avaliar a eficácia de instrumentos endodônticos rotatórios na modelagem de canais radiculares decíduos. O preparo do terço coronal da raiz foi maior usando limas rotatórias, afilando gradualmente no terço médio e apical do canal. Esse formato, causado pelo desenho específico das limas rotatórias, corresponde melhor à anatomia do canal radicular dos dentes decíduos. Já as limas manuais proporcionam um preparo coronal mais limitado, podendo dificultar uma obturação ideal dos canais. 
Por fim, o estudo de Tyagi et al., (2021 avaliou o tempo de instrumentação, a dor pós-operatória e os efeitos no comportamento da criança após pulpectomias com sistemas rotatórios e manuais. Não houve diferença significativa para o tempo médio do acesso endodôntico, entretanto, o tempo médio para o preparo biomecânico do canal foi significativamente maior naqueles preparados com limas manuais, com 40,02 minutos. A dor pré-operatória foi semelhante entre todos os pacientes, mas a dor após o procedimento foi consideravelmente maior quando realizado por limas manuais após as primeiras 6 horas, não ocorrendo mais diferenças após as 24 horas de pós-operatório. Nenhuma variação no comportamento das crianças foi observada, independente do tipo de lima utilizada.

Tabela 1 - Características e dados dos artigos selecionados.

\begin{tabular}{|c|c|c|}
\hline Autor/ Ano & Amostra & Principais dados encontrados \\
\hline $\begin{array}{l}\text { Barasuol et } \\
\text { al., 2021 }\end{array}$ & 88 participantes. & $\begin{array}{l}\text { O preparo químico-mecânico de canais } \\
\text { radiculares em crianças diminuiu em } 7,6 \\
\text { minutos com limas rotatórias em } \\
\text { comparação com as limas manuais. Outros } \\
\text { valores não tiveram diferenças significativas. }\end{array}$ \\
\hline $\begin{array}{l}\text { Boonchoo et } \\
\text { al., } 2020\end{array}$ & $\begin{array}{l}34 \text { crianças, sendo que } \\
18 \text { elementos dentários } \\
\text { foram tratados por limas } \\
\text { rotatórias e } 19 \text { por limas } \\
\text { manuais. }\end{array}$ & $\begin{array}{l}\text { A instrumentação dos canais foi } \\
\text { significativamente mais rápida quando } \\
\text { usadas as limas rotatórias, bem como o } \\
\text { preenchimento dos canais foi melhor } \\
\text { utilizando esta técnica. Entretanto, não } \\
\text { existem diferenças entre o sucesso do } \\
\text { tratamento das duas limas. }\end{array}$ \\
\hline
\end{tabular}

$\begin{array}{lll}\text { Govindaraju } & 45 \text { crianças. } & \text { A qualidade de obturação dos canais } \\ \text { et al., 2017 } & \text { dentários foi semelhante entre as limas } \\ & \text { manuais e rotatórias, porém, os canais } \\ & \text { mesiais tiveram qualidade melhor que os } \\ & \text { distais. As limas rotatórias apresentaram } \\ & \text { menor tempo de instrumentação em } \\ & \text { comparação às limas manuais. }\end{array}$

Jeevanandan 100 molares decíduos. e Thomas,

2018.

$\begin{array}{lll}\begin{array}{l}\text { Jeevanandan } \\ \text { et al., 2020 }\end{array} & 60 \text { participantes. } & \begin{array}{l}\text { Durante o intervalo de } 6 \text { a } 12 \text { horas após o } \\ \text { tratamento endodôntico foi relatado maior }\end{array} \\ & \text { intensidade de dor em pacientes tratados } & \\ & \text { com limas manuais. Após } 24 \text { horas do } \\ & \text { procedimento, não existiam mais diferenças } \\ & \text { em relação à dor. }\end{array}$

Canais instrumentados com limas manuais apresentaram maior abertura de diâmetro após a preparação dos canais. Instrumentos manuais apresentaram o maior tempo de preparação, quando comparado aos rotatórios.
Conclusões obtidas

As duas técnicas são consideradas seguras para o tratamento endodôntico em dentes decíduos, entretanto, só foram constadas diferenças entre o tempo de preparo químico-mecânico entre as limas.

As limas rotatórias possuem a vantagem de ter um menor tempo de instrumentação dos canais, simplificando o procedimento endodôntico em dentes decíduos, sendo uma boa alternativa ao uso das limas manuais.

O uso de instrumentos rotatórios para tratamentos endodônticos em dentes decíduos demanda menor tempo de preparo dos canais, influenciando positivamente o comportamento da criança dentro do consultório odontológico.

Instrumentações de canais com limas manuais causam mais dor pós-operatória do que as limas rotatórias. A dor é significativamente maior durantes as 12 primeiras horas após o procedimento.

Ocorreu menor intensidade de dor pós-operatória em canais tratados com limas rotatórias. A obturação ideal dos canais resulta em menor experiência de dor após o tratamento, sendo necessário outros estudos que suportem esses dados. 


\begin{tabular}{|c|c|c|}
\hline $\begin{array}{l}\text { Lakshmanan } \\
\text { et al., } 2020\end{array}$ & 45 crianças. & $\begin{array}{l}\text { O tempo médio de instrumentação foi menor } \\
\text { em tratamentos realizados com limas } \\
\text { rotatórias, com valor de } 75,6 \text { segundos. } \\
\text { Tratamentos realizados com limas rotatórias } \\
\text { tiveram canais com obturação ideal em } 60 \% \\
\text { dos casos, e os casos tratados com limas } \\
\text { manuais obtiveram sucesso em } 27 \% \text { dos } \\
\text { canais }\end{array}$ \\
\hline $\begin{array}{l}\text { Mehlawat et } \\
\text { al., } 2019\end{array}$ & 90 canais radiculares. & $\begin{array}{l}\text { O tempo médio da preparação biomecânica } \\
\text { dos canais foi de } 4,32 \text { em limas manuais, e } \\
3,54 \text { minutos em rotatórias. Sobre a eficácia } \\
\text { de limpeza dos canais, não houve diferenças } \\
\text { estatisticamente significativas entre os dois } \\
\text { tipos de lima. }\end{array}$ \\
\hline
\end{tabular}

Nair et al., 75 participantes.

2018

Pawar et al., 90 pacientes.

2021

$\begin{array}{lll}\begin{array}{l}\text { Ramazani et } 60 \text { molares decíduos. } \\ \text { al., 2016 }\end{array} & \begin{array}{l}\text { Os sistemas de limas rotatórias apresentaram } \\ \text { maior eficácia de limpeza e melhor } \\ \text { modelagem dos canais endodônticos. }\end{array} \\ & \begin{array}{l}\text { Também foram os sistemas com menor } \\ \text { tempo de instrumentação. }\end{array} \\ \text { Rathi et al., 20 pacientes. } & \text { Todas as técnicas de instrumentação com } \\ \mathbf{2 0 2 1} & \text { limas rotatórias causaram uma quantidade } \\ & \text { significativa de detritos extrusados. A } \\ & \text { eficácia de instrumentação foi considerada } \\ & \text { melhor nos terços coronal e, principalmente, } \\ & \text { no médio, sendo a região apical a de menor } \\ \text { eficiência de instrumentação. }\end{array}$
sintomatologia dolorosa durante as primeiras 24 horas do pós-operatório. Os pacientes que foram tratados com limas rotatórias não relataram experiência de dor.

O tempo de instrumentação de canais com limas rotatórias diminuíram em 20\% o tempo total do procedimento, em comparação com as limas manuais. A qualidade de obturação dos canais também foi superior entre as limas rotatórias.
O sistema de limas rotatórias forneceu maior qualidade de obturação dos canais radiculares, realizando a instrumentação em tempo médio menor que as limas manuais.
Instrumentos rotatórios oferecem qualidade de limpeza semelhante aos instrumentos manuais, realizando o procedimento em tempo muito menor. Portanto, as limas rotatórias possuem o potencial de substituir as limas manuais em tratamentos pediátricos.

A preparação químico-mecânica dos canais com limas rotatórias causa menos dor pós-operatória quando comparado aos sistemas de limas manuais.

\begin{tabular}{|c|c|c|}
\hline $\begin{array}{l}\text { Seema et al., } \\
2020\end{array}$ & 60 molares decíduos. & $\begin{array}{l}\text { As limas manuais e rotatórias apresentaram } \\
\text { uma quantidade média semelhante de } \\
\text { remoção de dentina. A conicidade do } \\
\text { preparo dos canais demonstrou que ambas as } \\
\text { limas produziam uma boa redução do canal } \\
\text { radicular. }\end{array}$ \\
\hline $\begin{array}{l}\text { Shah et al., } \\
2021\end{array}$ & 45 pacientes. & $\begin{array}{l}\text { Limas manuais possuíram um maior tempo } \\
\text { médio necessário para a instrumentação, } \\
\text { com } 27,87 \text { minutos e exibiram um maior } \\
\text { tempo de obturação dos canais, com uma } \\
\text { média de } 5,44 \text { minutos. Não houve } \\
\text { diferenças significativas entre as limas com } \\
\text { base na extensão do preenchimento do canal } \\
\text { e a presença ou ausência de vazios durante a } \\
\text { obturação. }\end{array}$ \\
\hline
\end{tabular}

Srinivas et 30 elementos dentários.
al., 2019

O preparo do terço coronal da raiz foi maior usando limas rotatórias, afilando gradualmente no terço médio e apical do canal.
O uso de limas endodônticas rotatórias resultou em procedimentos mais rápidos e com melhor qualidade de obturação dos canais nos dentes decíduos.

Limas rotatórias demonstraram boa conicidade do canal radicular. Os movimentos rotativos melhoraram a eficácia de limpeza desses canais, e ainda permitiram a diminuição do tempo de preparação dos canais.

Dentro das suas limitações, os sistemas de limas rotatórias causaram extrusão de detritos. A região apical das raízes foi a que menos apresentou eficácia de instrumentação.

Os sistemas de limas não apresentam diferenças entre o desgaste de dentina. A conicidade entre os sistemas também foi semelhante.

A eficácia do tempo de instrumentação e obturação dos canais é importante em se tratando de dentes decíduos, e as limas rotatórias foram melhores nesse aspecto. Logo, essas limas promoveram um procedimento com maior qualidade e chances de sucesso. As limas rotárias são opções mais seguras e eficientes que as manuais para uso pediátrico.

Limas rotatórias produzem canais com preparação mais cônica nos dentes decíduos, sendo considerado um formato ideal para facilitar a obturação dos canais. 


Tyagi et al., 75 pacientes.
$\mathbf{2 0 2 1}$
2021

\begin{tabular}{|c|c|c|}
\hline $\begin{array}{l}\text { Acharya et } \\
\text { al., } 2019\end{array}$ & $\begin{array}{l}60 \text { crianças de até } 12 \\
\text { anos. }\end{array}$ & $\begin{array}{l}100 \% \text { das crianças mostraram } \\
\text { comportamento positivo durante a } \\
\text { consulta odontológica na presença dos } \\
\text { pais. Já na ausência, o número passou para } \\
78,3 \% \text {. A presença dos pais é mais } \\
\text { influente durante os } 3 \text { a } 5 \text { anos. }\end{array}$ \\
\hline $\begin{array}{l}\text { Ahuja et al., } \\
2018\end{array}$ & $\begin{array}{l}30 \text { crianças acima dos } 4 \\
\text { aos } 7 \text { anos. }\end{array}$ & $\begin{array}{l}80 \% \text { das crianças com comportamento } \\
\text { negativo na primeira consulta mostraram } \\
\text { uma conduta positiva na segunda visita, } \\
\text { mesmo com a ausência dos pais. } 95,8 \% \\
\text { das crianças que apresentaram } \\
\text { comportamento positivo, continuaram com } \\
\text { a mesma postura com a ausência dos pais } \\
\text { na segunda consulta. }\end{array}$ \\
\hline
\end{tabular}

O tempo médio para o preparo biomecânico do canal foi significativamente maior naqueles preparados com limas manuais, com 40,02 minutos. A dor pré-operatória foi consideravelmente maior quando realizado por limas manuais após as primeiras 6 horas de procedimento.
O desempenho geral dos sistemas rotatórios foi considerado melhor do o manual. As limas rotatórias apresentaram menor tempo de instrumentação e obturação, bem como menor experiência com dor pós-operatória. Porém, o comportamento da criança não sofreu mudanças.

\begin{abstract}
Cadermatori 124 crianças entre 7 e $10 \quad 58,9 \%$ das crianças apresentaram et al., 2017 anos. comportamento não cooperativo e 54,1\% foram acompanhadas pelas mães. Crianças com a presença de mães ansiosas com o tratamento tiveram $52 \%$ mais prevalência de um comportamento não cooperativo durante extrações dentárias, que foi avaliada em $38 \%$ dos casos nas crianças acompanhadas.

$\begin{array}{ll}\text { Cox et al., } 90 \text { crianças entre } 4 \text { e } 12 & \text { Os dentistas informaram um } \\ \text { anos. } & \text { comportamento mais negativo com a } \\ & \text { presença dos pais durante o procedimento } \\ & \text { odontológico. As crianças mais novas } \\ & \text { (entre } 4 \text { e } 5 \text { anos) foram associadas a uma } \\ & \text { conduta mais negativa quando os pais } \\ & \text { estavam presentes no consultório } \\ & \text { odontológico. }\end{array}$
\end{abstract}

Desai et al., 300 pais com filhos em $96 \%$ das mães de crianças com 2 a 5 anos 2019 idade entre 2 e 13 anos. informaram preferir estar com os filhos durante o atendimento, sendo que essa porcentagem fica abaixo dos $90 \%$ conforme a idade das crianças aumenta. $100 \%$ dessas mães achavam que sua presença nas consultas tornariam as crianças mais cooperativas. Esses valores também diminuem conforme a idade das crianças aumenta.

$85 \%$ dos dentistas afirmaram que permitiria a presença dos pais durante $o$ tratamento de crianças com menos de 18 meses, $79 \%$ permitia em casos de crianças entre 18 e 25 meses e $63 \%$ em casos de crianças com 30 a 60 meses de idade. 53\% dos dentistas disseram que a presença dos pais foi útil para tratar crianças de 5 a 9 anos com comportamento negativo. $62 \%$ dos pais que estavam presentes durante a consulta disseram que seus filhos reagiriam favoravelmente às próximas consultas. E $23 \%$ dos pais que estavam
O pai deve auxiliar o comportamento da criança orientado pelo dentista, que precisa entender como os pais influenciam no comportamento das crianças para que se tenha bons resultados sobre o comportamento infantil.

O estudo demonstra a importância de separar os pais durante o atendimento para gerenciar o comportamento das crianças. Durante o tratamento odontológico pode se recomendar excluir a presença dos pais para eliminar muitos problemas relacionados ao comportamento.

A presença da mãe, principalmente as mães com ansiedade sobre o tratamento dos filhos, foi associado ao comportamento da criança durante a extração dentária.

As crianças apontaram não existir diferenças entre o tratamento odontológico realizado com ou sem a presença dos pais. Já os dentistas reportaram maiores dificuldades em diminuir a ansiedade da criança quando os pais estavam presentes.

Os pais afirmaram que sua presença teria um efeito positivo na cooperação de seus filhos. Eles podem dar apoio adequado, especialmente para crianças muito pequenas em situações novas e desafiadoras.

Existem razões que justifiquem a presença ou ausência dos pais no consultório odontológico. Existe uma tendência de os pais preferirem estar presentes durante as consultas dos filhos, principalmente os de menor idade. Excluir a presença dos pais sem que seja necessário nega a oportunidade de utilizar os pais como um reforço positivo para o comportamento inicial das crianças. 
ausentes informaram que seus filhos responderiam favoravelmente nas próximas sessões.

\begin{tabular}{|c|c|c|}
\hline $\begin{array}{l}\text { Pani et al., } \\
2016\end{array}$ & $\begin{array}{l}122 \text { crianças entre } 6 \text { e } 8 \\
\text { anos. }\end{array}$ & $\begin{array}{l}\text { A frequência cardíaca dessas crianças foi } \\
\text { aferida, constando que aquelas } \\
\text { acompanhadas dos pais apresentaram } \\
\text { frequências mais baixas que as crianças } \\
\text { desacompanhadas. As crianças } \\
\text { desacompanhadas eram menos ansiosas e } \\
\text { tinham comportamento mais positivo do } \\
\text { que as acompanhadas pelos pais. }\end{array}$ \\
\hline $\begin{array}{l}\text { Rodriguez et } \\
\text { al., } 2018\end{array}$ & $\begin{array}{l}105 \text { pais de crianças } \\
\text { tratadas. }\end{array}$ & $\begin{array}{l}36 \% \text { dos pais relataram ansiedade durante } \\
\text { o tratamento dos filhos. Os dentistas } \\
\text { relataram que do total de pais com } \\
\text { instrução para se manterem observadores } \\
\text { passivos, } 67,3 \% \text { permaneceram de fato } \\
\text { passivos, enquanto } 32,1 \% \text { dos não } \\
\text { instruídos foram considerados passivos. }\end{array}$ \\
\hline
\end{tabular}

$\begin{array}{lll}\begin{array}{l}\text { Sabbagh e } \\ \text { Sijini, 2020 }\end{array} & \begin{array}{l}283 \text { pais de crianças } \\ \text { atendidas em uma clínica } \\ \text { pediátrica da Arábia }\end{array} \\ \begin{array}{l}\text { Saudita. } \\ \text { Vasiliki }\end{array} & \text { et } & \begin{array}{l}100 \text { crianças de } 4 \text { a } 12 \\ \text { anos. }\end{array} \\ \text { al., 2016 } & \end{array}$

$24 \%$ dos pais preferiram não estar presentes durante $\mathrm{o}$ atendimento das crianças, e $76 \%$ não aceitaram a separação. $42,9 \%$ dos pais presentes durante a consulta informaram preferir estarem separados dos filhos que apresentaram comportamento negativo. E $33,3 \%$. Preferiram naquelas crianças com comportamento positivo.
A presença dos pais durante o atendimento odontológico reduz as manifestações fisiológicas das crianças durante suas primeiras restaurações dentárias.

Uma instrução para que os pais sejam observadores passivos é eficaz para auxiliar o trabalho do dentista. Essa instrução não afetou a satisfação dos pais em relação aos cuidados dentários com a criança.
Crianças acompanhadas dos pais apresentaram um aumento do desconforto entre as três primeiras consultas consecutivas, não ocorrendo essa diferença de incômodo quando as crianças estavam sozinhas.
A maioria dos pais não preferiu ficar separado dos filhos durante o atendimento odontológico por causa da segurança e proteção das crianças.
A presença dos pais durante o tratamento dentário não influencia no comportamento das crianças. Os pais e os dentistas observaram uma piora no comportamento das crianças quando os pais estavam ausentes. As crianças parecem ter uma experiência mais positiva na presença dos pais.

Fonte: Elaborado pelos autores (2021).

\section{Discussão}

A utilização de sistemas de limas rotatórias para tratamentos endodônticos em dentes decíduos vem sendo bastante discutida ao longo do tempo, principalmente nos últimos cinco anos. Em geral, os estudos abordam as vantagens na utilização desses sistemas de limas, comparando-os aos sistemas padrões na odontopediatria de limas manuais. A principal questão tomada como a maior vantagem das limas rotatórias diz respeito ao tempo clínico de instrumentação dos canais radiculares. Todos os estudos avaliados neste trabalho demonstraram que o uso das limas rotatórias diminui consideravelmente o tempo de instrumentação dos canais de dentes decíduos, quando comparados ao tempo de instrumentação das limas manuais. Além disso, Shah et al., (2021) relataram que as limas rotatórias também exibiram um menor tempo de obturação dos canais, possivelmente devido à redução do tempo de instrumentação, resultando em menor fadiga do profissional que está realizando o tratamento. Esses dados diferem do estudo de Boonchoo et al., (2020), que não relataram diferenças entre o tempo de obturação dos canais tratados com limas manuais e rotatórias, sendo um tema que merece maiores discussões para estabelecer essa correlação.

Essa redução do tempo operatório apresentou variações entre os estudos, provavelmente devido ao fato de os estudos utilizarem sistemas rotatórios diferentes. Enquanto Barasoul et al., (2021) descreveram uma média de 7,6 minutos a menos de tempo clínico para a instrumentação dos canais, Boonchoo et al., (2020) relataram uma diminuição de 4 minutos do 
procedimento de instrumentação. Já Pawar et al., (2021) indicaram uma redução de $20 \%$ do tempo entre os sistemas de limas analisados. Apesar dessas diferenças, a diminuição do tempo operatório em todos os casos foi considerada estatisticamente significante, sendo um fator a ser levado em consideração para a escolha do método de tratamento para as crianças, apesar de Barasoul et al., (2021) e Tyagi et al., (2021) terem relatado que a diminuição do tempo operatório não afetou o comportamento da criança dentro do consultório, mas poderia aumentar a longo prazo a sua cooperação em relação aos tratamentos odontológicos.

O principal fator de sucesso dos tratamentos endodônticos consiste em um bom preparo biomecânico dos canais radiculares, sendo necessário avaliar se a diminuição do tempo de instrumentação com as limas rotatórias pode influenciar na qualidade de preparo dos canais (Toline et al., 2021). Ficou claro que as limas rotatórias não são inferiores às manuais em relação ao preparo mecânico dos canais, apresentando uma boa modelagem dos canais em dentes decíduos. (Ramazani et al., 2016; Seema et al., 2020). Srinivas et al., (2019) ainda relaram que as limas rotatórias, quando comparadas às limas manuaus, apresentaram uma melhor qualidade do preparo do terço coronal da raiz, afilando gradualmente no terço médio e apical do canal. Essa modelagem resultou em um preparo de canal com um formato específico que se assemelhou melhor à anatomia do canal radicular dos dentes decíduos. Em conflito com os demais estudos, Jeevanandan e Thomas, (2018) demonstraram que as limas manuais apresentaram maior abertura de diâmetro após a preparação dos canais, facilitando as demais etapas da pulpectomia. Apesar disso, as limas rotatórias foram consideradas eficientes para o tratamento de canal em dentes decíduos. Esses resultados demonstram a eficácia dos sistemas rotatórios na modelagem dos canais.

Um outro ponto a ser abordado sobre o preparo mecânico das limas rotatórias diz respeito à eficácia de limpeza dos canais radiculares dos dentes decíduos. Mehlawat et al., (2019) e Ramazani et al., (2016) evidenciaram uma boa qualidade de limpeza dos canais tratados com sistemas rotatórios, sendo semelhante aos manuais. Entretanto, o estudo de Rathi et al., (2021) informou que as limas endodônticas expelem uma certa quantidade de detritos para a região periapical dos canais, porém, esses detritos devem ser mínimos, pois pode causar efeitos tóxicos no germe dentário permanente. Assim, além da eficiência de limpeza, um instrumento endodôntico deve possuir a capacidade de limitar a extrusão dos detritos para a região apical. Nesse sentido, esse estudo mostrou que as limas rotatórias causaram uma quantidade significativamente maior de detritos extrusados apicalmente em comparação às limas manuais, acarretando o chamado "efeito taper lock", onde a lima fica envolvida na região coronal do canal e não permite que os detritos da instrumentação saiam do canal, empurrando-os para a porção apical.

O preparo mecânico dos canais radiculares influencia diretamente na sua qualidade da obturação, sendo um fator essencial a ser avaliado durante o tratamento endodôntico com limas rotatórias. Os estudos que abordaram a qualidade da obturação em canais de dentes decíduos mostraram que o preenchimento desses canais obteve melhor qualidade quando foram tratados através de instrumentos endodônticos rotatórios, quando comparados aos instrumentos manuais. (Boonchoo et al., 2020; Govindaraju et al., 2017; Jeevanandan et al., 2021; Lakshmanan et al., 2020; Pawar et al., 2021; Shah et al., 2021).

Por sua vez, a qualidade de obturação está intimamente ligada à experiência de dor pós-operatória. A presença de dor após tratamentos endodônticos também varia de acordo com o nível de ansiedade do paciente, estado de dor pré-operatória, limiar de dor individual, restos de tecido pulpar, irritação mecânica e química dos tecidos periapicais e a extrusão apical dos detritos infectados da raiz. (Jeevanandan et al., 2021). Com base nisso, resta saber se as limas rotatórias acarretaram em uma menor intensidade de dor após os tratamentos de pulpectomia. Os estudos analisados relataram que canais tratados com limas manuais, quando comparado às limas rotatórias, tiveram maior porcentagem de casos com dor moderava a severa, principalmente durante as seis primeiras horas após o tratamento endodôntico. Também ficou comprovado que a dor possuía uma duração maior nesses casos, em torno de 24 horas após o procedimento operatório. Ao fim desse período, as dores tendem a diminuir, não ocorrendo mais diferenças entre tratamentos com limas manuais ou rotatórias. (Fonseca et al., 2020; Barasoul et al., 2021; Jeevanandan et al., 2020; Jeevanandan et al., 2021; Nair et al., 2018; Tyagi et al., 2021). 


\section{Conclusão}

Tratamentos endodônticos em dentes decíduos utilizando sistemas de limas rotatórias proporcionaram um preparo biomecânico mais rápido dos canais radiculares, acarretando em consultas mais curtas, que melhoram o conforto do paciente, e a longo prazo podem aumentar a cooperação e confiança das crianças em relação aos tratamentos odontológicos, diminuindo as dificuldades dos dentistas na abordagem endodôntica pediátrica. Além disso, as limas rotatórias também demonstraram uma boa modelagem e alta eficiência de limpeza dos canais radiculares, o que acarretou em uma alta taxa raízes obturadas de modo adequado. Por fim, as limas rotatórias ainda demonstraram causar uma menor quantidade de casos de dor pós-operatória, quando comparado às limas manuais. Esses achados afirmam que o uso de instrumentos rotatórios no tratamento endodôntico em crianças é eficaz, rápido, seguro, e com maior conforto para as crianças, sendo uma opção viável e um bom substituto para as limas manuais, mas que ainda necessita de maiores estudos para corroborar esses resultados de maneira segura.

\section{Referências}

Ahmed, H. M. A. (2013). Anatomical challenges, electronic working length determination and current developments in root canal preparation of primary molar teeth. International Endodontic Journal, 46 (1), 1011-1022.

Barasuol, J. C., Massignan, C., Bortoluzzi, E. A., Cardoso, M., \& Bolan, M. (2021). Influence of hand and rotary files for endodontic treatment of primary teeth on immediate outcomes: Secondary analysis of a randomized controlled trial. Int J Paediatr Dent, 31 (1), $143-151$.

Boonchoo, K., Leelataweewud, P., Yanpiset, K., \& Jirarattanasopha, V. (2020). Simplify pulpectomy in primary molars with a single-file reciprocating system: a randomized controlled clinical trial. Clinical Oral Investigations, 24 (1), 2683-2689.

Chauhan, A., Saini, S., Dua, P., \& Mangla, R. (2019). Rotary Endodontics in Pediatric Dentistry: Embracing the New Alternative. International Journal of Clinical Pediatric Dentistry, 12 (5), 460-463.

Chugh, V. K., Patnana, A. K., Chugh, A., Kumar, P., Wadhwa, P., \& Singh, S. (2021). Clinical differences of hand and rotary instrumentations during biomechanical preparation in primary teeth-A systematic review and meta-analysis. Int J Paediatr Dent., 31 (1), $131-142$.

George, S., Anandaraj, S., Issac, J. S., John, S. A., \& Harris, A. (2016). Rotary endodontics in primary teeth - A review. The Saudi Dental Journal, 28 (1), 1217.

Govindaraju, L., Jeevanandan, G., \& Subramanian, E. M. G. (2017). Comparison of quality of obturation and instrumentation time using hand files and two rotary file systems in primary molars: A single-blinded randomized controlled trial. European Journal of Dentistry, 11 (3), $376-379$.

Hermont, A. P., Zina, L. G., Silva, K. D., Silva, J. M., \& Martins-Júnior, P. A. (2021). Revisões integrativas: conceitos, planejamento e execução. Arq Odontol, 57 (1), 3-7.

Jeevanandan, G., \& Thomas, E. (2018). Volumetric analysis of hand, reciprocating and rotary instrumentation techniques in primary molars using spiral computed tomography: An in vitro comparative study. European Journal of Dentistry, 12 (1), 21-26.

Jeevanandan, G., Ravindran, V., Subramanian, E. M. G., \& Kumar, A. (2020). Postoperative Pain with Hand, Reciprocating, and Rotary Instrumentation Techniques after Root Canal Preparation in Primary Molars: A Randomized Clinical Trial. International Journal of Clinical Pediatric Dentistry, 13 (1), 21-26.

Jeevanandan, G., Govindaraju, L., Subramanian, E. M. G., \& Priyadarshini, P. (2021). Comparative Evaluation of Quality of Obturation and Its Effect on Postoperative Pain between Pediatric Hand and Rotary Files: A Double-blinded Randomized Controlled Trial. International Journal of Clinical Pediatric Dentistry, 14 (1), 88-96.

Lakshmanan, L., Mani, G., Jeevanandan, G., Ravindran, V., \& Erulappan Muthu Ganapathi, S. (2020). Assessing the quality of root canal filling and instrumentation time using kedo-s files, reciprocating files and k-files. Braz Dent Sci., 23 (1), 1-7.

Manchanda, S., Sardana, D., \& Yiu, C. K. Y. (2020). A systematic review and meta-analysis of randomized clinical trials comparing rotary canal instrumentation techniques with manual instrumentation techniques in primary teeth. International Endodontic Journal, 53 (1), $333-353$.

Mehlawat, R., Kapoor, R., Gandhi, K., Kumar, D., Malhotra, R., \& Ahuja, S. (2019). Comparative evaluation of instrumentation timing and cleaning efficacy in extracted primary molars using manual and NiTi rotary technique - Invitro study. Journal of Oral Biology and Craniofacial Research, 9 (1), $151-155$.

Moher, D., Liberati, A., Tetzlaff, J., Altman, D. G., \& The PRISMA Group. (2009). Preferred Reporting Items for Systematic Reviews and Meta-Analyses: The PRISMA Statement. Plos Medicine, 6 (7), 1-6.

Nair, M., Jeevanandan, G., Vignesh, R., \& Subramanian, E. M. G. (2018). Comparative evaluation of post-operative pain after pulpectomy with K-files, Kedo$\mathrm{S}$ files and MTwo files in deciduous molars -a randomized clinical trial. Braz Dent Sci, 21 (4), 411-417.

Natchiyar, N., Asokan, S., Priya, P. R. G., \& Kumar, T. D. Y. (2021). Comparison of Clinical and Radiographic Success of Rotary with Manual Instrumentation Techniques in Primary Teeth: A Systematic Review. International Journal of Clinical Pediatric Dentistry, 14 (1), 8-13. 
Research, Society and Development, v. 10, n. 16, e495101624050, 2021

(CC BY 4.0) | ISSN 2525-3409 | DOI: http://dx.doi.org/10.33448/rsd-v10i16.24050

Pawar, B., Pawar, A. M., Bhardwaj, A., Wahjuningrum, D. A., Rahardjo, A. K., Luke, A. M., Metzger, Z., \& Kfir, A. (2021). Effect of Adaptive, Rotary, and Manual Root Canal Instrumentation in Primary Molars: A Triple-Armed, Randomized Controlled Clinical Trial. Biology, 42 (1), 1-11.

Ramazani, N., Mohammadi, A., Amirabadi, F., Ramazani, M., \& Ehsani, F. (2016). In vitro investigation of the cleaning efficacy, shaping ability, preparation time and file deformation of continuous rotary, reciprocating rotary and manual instrumentations in primary molars. JODDD, 10 (1), 49-56.

Rathi, N., Jain, S., Thosar, N., Baliga, S., Ahmed, F., \& Metha, J. (2021). Comparative Evaluation of Cleaning Efficiency and Apical Extrusion of Debris Using Two Pediatric Rotary Endodontic Files: An In Vitro Study. International Journal of Clinical Pediatric Dentistry, 14 (2), 196-200.

Seema, T., Ahammed, H., Parul, S., \& Cheranjeevi, J. (2020). Comparative Evaluation of Dentin Removal and Taper of Root Canal Preparation of Hand K File, ProTaper Rotary File, and Kedo S Rotary File in Primary Molars Using Cone-beam Computed Tomography. International Journal of Clinical Pediatric Dentistry, 13 (4), 332-336.

Shah, H. S., Patil, V. M., Kamath, A. P., \& Mathur, A. A. (2021). Comparative Evaluation of Instrumentation Time, Obturation Time, and Radiographic Quality of Obturation Using Two Rotary Systems and Manual Technique for Primary Molar Pulpectomies - In vivo Study. Contemporary Clinical Dentistry, $12(1), 55-62$

Srinivas, A., Jeevanandan, G., Govindaraju, L., \& Subramanian, E. M. G. (2019). Comparative Evaluation of The Efficacy Of Rotary file system (Kedo-S) Vs Hand K-Files In Root Canal Preparation Of Primary Teeth Using Cone Beam Computed Tomography (CBCT) - An In Vitro Study. Braz Dent Sci., 22 (2), 197-202.

Tyagi, R., Khatri, A., Kalra, N., \& Sabherwal, P. (2021). Comparative Evaluation of Hand K-flex Files, Pediatric Rotary Files, and Reciprocating Files on Instrumentation Time, Postoperative Pain, and Child's Behavior in 4-8-year old Children. International Journal of Clinical Pediatric Dentistry, 14 (2), 201206. 